\title{
FOTOELECTROQUÍMICA \\ EN SISTEMAS NANOESTRUCTURADOS: \\ UNA DISCUSIÓN DESDE SUS \\ LÍMITES NATURALES
}

\section{PHOTOELECTROCHEMISTRY IN NANOSTRUCTURED SYSTEMS: A DISCUSSION FROM THEIR NATURAL LIMITATIONS}

\author{
Dalia Leon $^{1,2}$, Daniel Torres ${ }^{1}$, Alberto Maimone ${ }^{2,3}$, \\ Franco M. Cabrerizo ${ }^{4}$, \\ Lorean Madriz ${ }^{1,5^{*}}$ \& Ronald Vargas ${ }^{1,4}$ *
}

Recibido: 31 de octubre 2020 / Aceptado: 18 de marzo 2021

DOI: $10.26807 /$ ia.vi.197

Palabras claves: Fotocorriente; fotoluminiscencia; portadores minoritarios; recombinación; semiconductores.

Keywords: Minority carriers; photocurrent;photoluminescence;

recombination; semiconductors.

1Universidad Simón Bolívar, Departamento de Química, Caracas, Venezuela. (loreanmmr@gmail. com)

2 Fundación Instituto de Ingeniería para el Desarrollo Tecnológico, Centro de Tecnología de Materiales, Caracas, Venezuela. (dalialeonchaparro@gmail.com)

3 Universidad Simón Bolívar, Departamento de Ciencias de los Materiales, Caracas, Venezuela. (al bertomaimone@gmail.com)

4 Universidad Nacional de San Martín, Consejo Nacional de Investigaciones Científicas y Técnicas (CONICET), Instituto Tecnológico de Chascomús. Chascomús, Argentina. (\#Dirección actual de RV. *Correspondencia: RV: ronaldvargas@usb.ve, ronaldvargas@intech.gov.ar).

5 Universidad Nacional de La Plata, Consejo Nacional de Investigaciones Científicas y Técnicas (CONICET), Instituto de Investigaciones Fisicoquímicas Teóricas y Aplicadas. La Plata, Argentina. (\#Dirección actual de LM. *Correspondencia: LM: loreanmmr@gmail.com, loreanmadriz@inifta. unlp.edu.ar) 


\section{RESUMEN}

Luego de reconocer que la respuesta fotoelectroquímica depende de la yuxtaposición de fenómenos de transferencia y de recombinación de las cargas fotogeneradas, y que dichos procesos representan límites naturales al comportamiento experimental, se discuten algunos aspectos fisicoquímicos que determinan el desempeño de una interfase semiconductor | electrolito, considerando específicamente la situación de un fotoánodo nanoestructurado. Se tomó como caso de estudio la relación entre la respuesta experimental de transferencia electrónica y la recombinación en nanotubos de $\mathrm{TiO}_{2}$, presentando estos una modificación en la relación de fases anatasa y rutilo. Mediante el análisis de la respuesta potenciodinámica a elevados sobrepotenciales en relación a la teoría de Gärtner, además de la cuantificación del tiempo de vida de portadores debido al decaimiento fotoluminiscente a circuito abierto, resultó posible ilustrar la relación cualitativa entre la cinética de transferencia de carga y la desactivación radiativa, procesos que, siendo opuestos, determinan la respuesta fotoelectroquímica de estos fotoánodos nanoestructurados.

\section{ABSTRACT}

The physicochemical aspects behind the performance of a semiconductor | electrolyte interphase are discussed, addressing the fact that the photoelectrochemical response depends on the juxtaposition of two fundamental phenomena: charge-carriers transfer and recombination. Such phenomena define the natural limits of the experimental behavior. Considering $\mathrm{TiO}_{2}$ nanotubes as a special case of study to illustrate the photoelectrochemical behavior of nanostructured electrodes, the analysis of the potentiodynamic response at high overpotentials in relation to Gärtner's theory shows how the incorporation of a different phase (rutile into anatase) can modulate the experimental response. The quantification of the lifetime of carriers with to photoluminescent decays at open circuit illustrates how the relationship between the kinetics of charge transfer and recombination determines the natural limitations of nanostructured photoanodes performance. 


\section{INTRODUCCIÓN}

La fotoelectroquímica está relacionada con las reacciones redox inducidas por luz. Una situación experimental de interés resulta cuando estas reacciones ocurren en un electrodo constituido por un material semiconductor, por lo que, al incidir una radiación de energía igual o mayor a la brecha de bandas (Eg) se promueven electrones desde la banda de valencia (EBV) hacia la banda de conducción (EBC). Los desarrollos actuales relacionados con la electroquímica de semiconductores son numerosos, destacándose los relacionados a conversión de energía en celdas solares (Kim, 2020), tratamiento de aguas mediante fotoelectroquímica (Garcia-Segura \& Brillas, 2017; Vargas et al., 2020), celdas de combustibles fotocatalíticas (Lianos, 2017), materiales foto y electroluminiscentes (Gutiérrez, 2020), sensores y biosensores (Kokkinos \& Economous, 2020) y generación de combustibles solares como hidrógeno (Kumaravel, 2019). Los conceptos fisicoquímicos desarrollados desde la década de los 60s han sido fundamentales para explicar el comporta- miento de estos sistemas (Peter, 2013). Sin embargo, para abordar los nuevos retos en ciencia y tecnología, los avances deben ser instrumento para la generación de nuevo conocimiento, especialmente en la comprensión del rol de las nanoestructuras. Antes de iniciar una discusión sobre el efecto de las nanoestructuras en los sistemas fotoelectroquímicos, se ilustrará de forma breve un marco conceptual de los fenómenos que tienen lugar en una interfase iluminada, semiconductor | electrolito. Si el lector desea abordar revisiones o desarrollos más detallados sobre los fundamentos de dichas temáticas, se recomienda revisar las respectivas obras clásicas de Sato y Memming (Sato, 1998; Memming, 2015).

\section{Un primer mapa conceptual: Aspec- tos básicos de la respuesta fotoelec- troquímica}

Al iluminar un semiconductor tipo $n$, los electrones pueden ser extraídos como fotocorriente $\left(i_{\mathrm{FC}}\right)$ mediante un circuito externo, en cuyo caso la deficiencia de electrones o "huecos" 
como portadores minoritarios en la banda de valencia se transportan hasta la interfase para oxidar a una especie donadora presente en el electrolito. La transferencia de carga se completa cuando ocurre la reducción de algún aceptor de electrones en el electrodo secundario. La intensidad de la iluminación $(I)$ y el potencial $(E)$ establecen una desviación continua del equilibrio, por lo que el perfil de las energías de las bandas se considera modificado, esto en relación a la situación de equilibrio o cuando el potencial de las bandas es plano $\left(E_{\mathrm{BP}}\right)$ (Memming, 2015). En la Figura 1 se presenta una ilustración de los procesos fotoelectroquímicos que tienen lugar en la interfase.

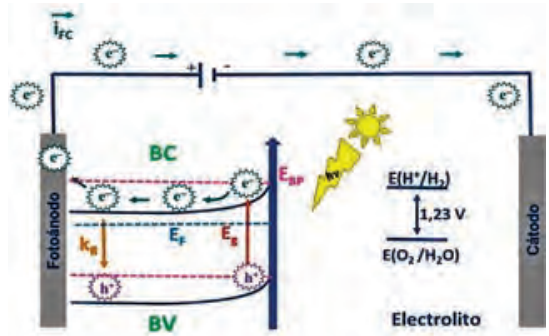

Figura 1. Procesos fotoelectroquímicos que tienen lugar en la interfase

La limitante más reconocida del proceso descrito es la recombinación de portadores, la cual da lugar a la disipación de energía $\left(k_{\mathrm{R}}\right)$, producto de la recombinación del electrón excitado con el hueco. A mayor gradiente de potencial electroquímico, los electrones fotogenerados se extraen con mayor eficiencia, mientras se define un delicado balance entre los flujos de transferencia electrónica y recombinación en la interfase semiconductor | electrolito, siendo las propiedades ópticas y eléctricas del material, determinantes en la generación de fotocorriente debida a los procesos redox (Gerischer, 1990).

El objetivo del presente trabajo es discutir e ilustrar aspectos relevantes de la fotoelectroquímica basada en nanoestructuras, para ello se comentará la fenomenología que define la condición de referencia ideal y las desviaciones de estas interfases, además de plantear la importancia del estudio dinámico de las mismas. Al ser una respuesta fenomenológica vale la pena preguntarse: ¿cómo discriminar efectos originados en la modificación sistemática de nanomateriales? y ¿cómo la interpretación de las medidas en nanoma- teriales se puede dirigir hacia una mejor com- 
prensión de la teoría fotoelectroquímica? La respuesta involucra diversas dimensiones, las cuales tocan transversalmente las condiciones de síntesis y la fisicoquímica de la señal electroquímica. Por tanto, elucidar diferencias en los casos límite, como los esperados a altos y bajos potenciales en relación al potencial de banda plana, puede resultar ilustrativo para sumar peso a una hipótesis que diferencie el motivo de los cambios observados debido al nanomaterial. En todo caso, la discusión de los parámetros cinéticos como elementos de comparación vuelve a ser clave para descubrir y sistematizar el comportamiento experimental.

\section{Postulados de una situación ideal: Aspectos fenomenológicos de la teoría de Gärtner}

La teoría de Gärtner implica conceptos fundamentales, los cuales vinculan el flujo de portadores minoritarios con las condiciones energéticas definidas por la intensidad de la luz y el potencial. En general, luego que la radiación defina un perfil de extinción en el electrodo: $G(\mathrm{x})$, la respuesta experimental dependerá de las propiedades características del semiconductor, como la densidad de portadores $\left(N_{D}\right)$, el coeficiente de absorción de radiación $(\boldsymbol{\alpha})$ y la longitud mínima de transporte de portadores (Lp), esto en la región de carga espacial (W) (Gärtner, 1959). La Figura 2 es una representación esquemática de la situación ideal considerada por Gärtner.

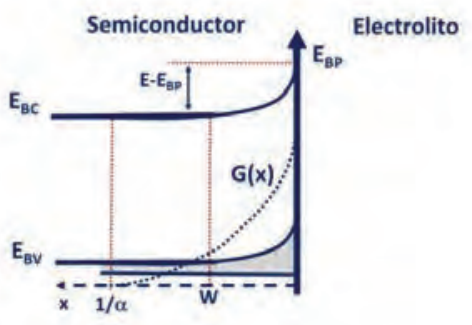

Figura 2. Diagrama energético según la situación ideal considerada por Gärtner

Los postulados fundamentales del modelo ideal son:

i.- La velocidad de generación de portadores depende del flujo de fotones y el coeficiente de absorción del semiconductor,

ii.- todos los portadores generados dentro de la región de carga espacial alcanzan la superficie al recorrer una longitud característica, 
iii.- una vez en la interfase, la fotocorriente es sostenida por un proceso redox con una velocidad de transferencia muy rápida, iv.- no hay pérdidas por recombinación.

La ecuación 1 representa el máximo teórico posible para la respuesta fotoelectroquímica en un semiconductor predicha por la densidad de corriente de Gärtner (jG), donde lo representa la intensidad de luz en la superficie (Gärtner, 1959).

$$
j_{G}=I_{0}\left[1-\frac{e^{-(\alpha W)}}{1+\alpha L_{p}}\right]
$$

siendo $\mathrm{W}$ definido por el potencial según la teoría de Mott y Schottky:

$$
W=\left[\frac{2 \varepsilon \varepsilon_{0}\left(E-E_{B P}\right)}{e_{0} N_{D}}\right]^{\frac{1}{2}}
$$

donde: $\varepsilon$ y $\varepsilon_{0}$ son las permisividades del semiconductor y del vacío, respectivamente, y eo la carga elemental.

El planteamiento es fenomenológico, siendo una condición clave para su implementación, la obtención de datos a potenciales mucho mayores que el valor de equilibrio, donde los postulados de Gärtner se satisfacen. Además, en el caso de semiconductor tipo $\mathrm{p}$ la situación es análoga, resaltando que los portadores minoritarios son los electrones y la corriente en la interfase es sostenida por un proceso de reducción (Memming, 2015).

Como ya se ha mencionado, la condición de equilibrio equivale al potencial de banda plana, por lo que determinar su valor resulta ventajoso para definir criterios para una apropiada evaluación. Su valor suele ser determinado usando mediciones de capacitancia diferencial de la zona de carga espacial $\left(C_{S}\right)$, y correlacionando estos datos con la teoría de Mott y Schottky (Ec. 3) (Memming, 2015).

$$
\frac{1}{C_{S}^{2}}=\left(\frac{2}{\varepsilon \varepsilon_{0} A^{2} e_{0} N_{D}}\right) E+\left(E_{B P}-\frac{k_{B} T}{e_{0}}\right)
$$

donde $A$ es el área, $k_{\mathrm{B}}$ la constante de Boltzmann y $T$ la temperatura. Un gráfico de $C_{S}^{-2}$ vs. E permitirá obtener $N_{\mathrm{D}}$ y $E_{\mathrm{BP}}$ por comparación con la región lineal. La determinación experimental toma en cuenta de forma implícita los efectos estructu- 
rales del electrodo. Para semiconductores tipo n se tiene que la pendiente del gráfico $C_{S}^{-2}$ vs. $E$ es positiva y $E_{\mathrm{BP}}=E_{\mathrm{BC}}$; mientras que, para semiconductores tipo $\mathrm{p}$ se define una pendiente negativa y $E_{\mathrm{BP}}=E_{\mathrm{BV}}$. Además, en cualquier caso, se cumple que: $E_{\mathrm{g}}=E_{\mathrm{BV}}-E_{\mathrm{BC}}$, por lo que, usar el valor de Eg determinado experimentalmente por métodos espectroscópicos permite estimar la posición de cada banda de energía del electrodo semiconductor.

En otro sentido, se debe destacar que Reichman incorporó al análisis de Gärtner, las modificaciones apropiadas para considerar efectos de recombinación, el aspecto clave se resumió en el balance diferencial de portadores, así como en su condición de contorno (Reichman, 1980).

\section{Desviaciones típicas de la interpre- tación fenomenológica de Gärtner: Dopaje y nanoestructuras}

En semiconductores con bajo o moderado dopaje, la capacitancia diferencial es controlada por los portadores de carga acumulados en la zona de carga espacial (Peter et al., 2018). A partir de entonce, se fijan los niveles energéticos del borde de las bandas, trayendo como consecuencia que los cambios en el potencial aplicado afecten principalmente a la zona de carga espacial (W, ver Ec. 2); mientras que la caída de potencial a través de las capas de Helmholtz y la difusa permanece constante. En las circunstancias descritas, la teoría de transferencia electrónica de Butler y Volmer no se puede aplicar, ya que la energía de activación para la transferencia de energía sería independiente del potencial. El hecho de que la fotocorriente aumente en función del potencial del electrodo no implica que se incremente la velocidad de transferencia de carga en la interfase; esto suele ser el resultado de una disminución en la cinética de recombinación (Gerischer, 1990; Peter et al., 1997; Vargas et al., 2020).

La condición donde se fija el nivel de Fermi (EF) también es posible, especialmente para densidades de estado superficiales superiores al $1 \%$ en relación a la densidad del átomo dopante en la superficie. Luego, los cambios en el potencial aplicado se pueden desarrollar en la capa de 
Helmholtz (Sato, 1998; Memming, 2015). En dicha situación, la capacitancia total $\left(C_{\mathrm{T}}\right)$ vendrá definida por la contribución en serie de las capacitancias de la zona de carga espacial $\left(C_{S}\right)$ y la de Helmholtz $\left(C_{H}\right)$ : $C_{\mathrm{T}^{-1}}=C_{\mathrm{S}^{-1}}+C_{\mathrm{H}^{-1}}$ (Monllor-Satoca et al., 2020).

En general, el dopaje, las diferentes fases cristalinas, estados adsorbidos y los defectos del material, introducen modificaciones a la densidad de estados electrónicos, los cuales en nanoestructuras se manifiestan entre las bandas de valencia y de conducción; estos defectos conocidos como estados superficiales afectan la dinámica de la interfase (Berger et al., 2012; Bisquert, 2017). En estos casos, $C_{S}$ se relaciona con la capacitancia intrínseca de la película fotoactiva semiconductora o capacitancia química $(C \mu)$, la cual suele ser mucho menor que $C_{\mathrm{H}}$ y domina el comportamiento electroquímico final (Bisquert, 2003). La capacitancia química depende de las características geométricas de la película nanoestructurada, así como, de la variación de la densidad de estados poblados con el nivel de Fermi (EF) (Bisquert, 2003; Monllor-Satoca et al., 2020). En general, las contribuciones vienen de la dinámica de estados en la banda de conducción, estados superficiales y estados profundos (Monllor-Satoca et al., 2020). En todo caso, se resaltará que una forma de modificar la densidad de estados de un semiconductor, tienen tiene que ver con el dopaje, la formación de nuevas fases cristalinas y su síntesis a escala nanométrica. El estudio de estos fenómenos se puede realizar empleando técnicas de espectroscopia de impedancia electroquímica (EIS, por sus siglas en inglés), y analizando la zona capacitiva de la voltametría cíclica en oscuridad (Bertolucci et al., 2013; Bisquert, 2017). En la literatura reciente se puede consultar una síntesis sobre la respuesta electroquímica de óxidos semiconductores nanoestructurados y capas compactas, donde se discuten fundamentos, así como detalles de estructura electrónica de electrodos nanoporosos: estados superficiales y profundos; todo ello enfatizando la naturaleza de los diferentes fenómenos capacitivos que tiene lugar en electrodos semiconductores (Monllor-Satoca et al., 2020). 
En relación al efecto de nanoestructura, el grupo de Peter planteó un análisis idealizado, el cual demuestra que, al tener en cuenta únicamente las consideraciones geométricas, se logra justificar la mejora de las eficiencias cuánticas para la transformación de energía luminosa usando electrodos nanoestructurados (Peter et al., 2018). Por ejemplo, se describieron los efectos de la geometría nanocilíndrica sobre el comportamiento de la capacitancia diferencial en función del potencial, permitiendo discutir el caso límite de agotamiento completo en términos de la capacidad geométrica residual en la base de los nanocilindros. Dado que las nanoestructuras generalmente dejan áreas del sustrato expuestas, para considerar estos efectos en la respuesta electroquímica, resulta necesario tomar en cuenta la capacitancia en paralelo asociada con la fracción de superficie descubierta. Estos argumentos permiten comprender de forma intuitiva los efectos de las nanoestructuras en la respuesta electroquímica; además, resultan diferentes a los comúnmente usados en la literatura, los cuales tienen que ver con dinámica y transporte de portadores en longitudes características, entre otros fenómenos no directamente proporcionales a los factores geométricos.

\section{La necesidad de evaluación diná- mica}

En los electrodos nanoestructurados el panorama es ciertamente más complejo e involucra cambios tanto en la población de estados superficiales como en la cinética de recombinación, dependiendo ambos procesos del potencial del electrodo (Bisquert, 2017; Monllor-Satoca et al., 2020). Además, el transporte de los portadores mayoritarios también requiere atención. El grupo de Fermín (Zhang et al. 2014) investigó este problema en el caso de nanoestructuras de $\mathrm{TiO}_{2}$ y de $\mathrm{ZnO}$ en las que la recombinación de la superficie fue suprimida por adición de un donador de electrones con reacción rápida con los huecos. En sus trabajos se investigó la población de estados superficiales profundos en electrodos nanoestructurados y las respuestas dinámicas de fotocorriente bajo iluminación superior a la brecha de bandas. Los experimentos de fotocorriente dinámica mostraron que el tiempo de ascenso 
característico de la corriente aumenta a medida que incrementa el potencial, esto a valores mayores que la región de inicio de carga de estados profundos. La ocupación de estos estados superficiales en condiciones estacionarias fue una fracción de la densidad de estados estimada a partir de respuestas voltamétricas. En presencia de un captador de huecos fuerte $\left(\mathrm{SO}_{3}{ }^{2-}\right)$, las curvas de fotocorriente frente al potencial mostraron un máximo en el rango en el que la población de estados superficiales depende del potencial aplicado. A un sobrepotencial suficientemente positivo donde se agotasen completamente los estados superficiales, la amplitud de la fotocorriente disminuyó fuertemente con el aumento de la frecuencia de perturbación de la luz. Estas ideas son particularmente relevantes cuando se considera el análisis sistemático de los transitorios de fotocorriente observados con electrodos nanoestructurados; o en el caso general de alguna señal dinámica, como las debidas a la perturbación periódica de la interfase con señales de luz o eléctricas (Peter et al., 1997; Peter, 2013).

\section{Caso de estudio: arreglos ordenados de nanotubos de $\mathrm{TiO}_{2}$}

Luego de haber discutido estas ideas, resulta pertinente ilustrar los planteamientos con algún sistema de interés. Los electrodos formados por anodización a altos campos tienen la ventaja de que la película activa se sintetiza in situ garantizando el contacto eléctrico con destacadas propiedades mecánicas. Además, se pueden obtener nanoestructuras ordenadas de diferente naturaleza y composición química, y la aplicación de un tratamiento térmico permite definir estructura cristalina y/o dopar al electrodo (Lee et al., 2014; Naranjo et al., 2017; Nevaréz-Martínez et al., 2018; Vargas et al., 2019). En este sentido, se seleccionaron arreglos nanotubulares de $\mathrm{TiO}_{2}$ como electrodos de prueba, explorándose, además, la modificación mediante el cambio de la relación de fases cristalinas a través de un tratamiento térmico, definiendo un primer electrodo $100 \%$ anatasa y otro $80 \%$ anatasa con 20 $\%$ de rutilo. La nueva fase modifica la densidad de estados y por tanto la respuesta electroquímica. En la literatura reciente se podrá consultar 
experimentación apropiada para la obtención de este tipo de materiales (Naranjo et al., 2017; Rueda et al.,
2018; Vargas et al., 2019; Leon et al., 2021).

\section{MATERIALES Y MÉTODOS}

A continuación se detallarán algunos aspectos sobre las condiciones experimentales para la obtención de los ensayos fotoelectroquímicos selectos, que servirán para ilustrar las diferencias de la respuesta fenomenológica desde sus límites naturales. Los detalles de la síntesis y caracterización de los electrodos basados en nanotubos de $\mathrm{TiO}_{2}$ pueden ser consultados en Maimone et al., 2015; Maimone, 2018; Leon, 2020 y Leon et al., 2021, además, en la Figura 3(A) se presenta la microscopía electrónica que permite visualizar la morfología de los materiales. Los ensayos sobre electrodos nanoestructurados fueron realizados en una celda de tres electrodos, provista con una ventana de cuarzo para permitir el paso de la luz UV-Visible, la cual fue modulada a través de una lámpara de xenón de $150 \mathrm{~W}$ desde un simulador solar (Newport). Mediante un potenciostato Autolab-PGSTAT30 se realizaron las mediciones potencios- táticas (cronoamperometría) y potenciodinámicas (voltametría de barrido lineal y de barrido cíclico, respectivamente), siempre midiendo respecto a un electrodo de referencia de $\mathrm{Ag}-\mathrm{AgCl}$ y usando un alambre de platino como electrodo secundario. La solución electrolítica estuvo constituida por soluciones acuosas de sulfato de sodio 0,1 M ( $\mathrm{Na}_{2} \mathrm{SO}_{4}$, Merck, 99,9 \%) y una mezcla de fosfato di-ácido de sodio $\left(\mathrm{NaH}_{2} \mathrm{PO}_{4} \cdot 2 \mathrm{H}_{2} \mathrm{O}\right.$, AnalaR, 99,0\%) con fosfato ácido de sodio $\left(\mathrm{Na}_{2} \mathrm{HPO}_{4} \cdot 7 \mathrm{H}_{2} \mathrm{O}\right.$, Mallinkrodt, 99,0 $\%$ ) como buffer pH 7. El agua desionizada fue purgada con gas $\mathrm{N}_{2}$ durante 20 min para remover el $\mathrm{O}_{2}$ disuelto. Las mediciones de fotoluminiscencia estacionaria y resuelta en el tiempo se realizaron usando un Fluoromax4 (HORIBA Jobin Yvon) y un single-photon-counting equipment FL3 TCSPC-SP (HORIBA Jobin Yvon) fluorometer, respectivamente. 


\section{RESULTADOS}

En la Figura 3(A) se presenta una microscopía electrónica de barrido donde se detalla la morfología del fotoánodo recién sintetizado. Las dimensiones características de este sistema se han reportado recientemente, y se resumen en los siguientes valores promedio: longitud de $568( \pm$ 60) nm, diámetro de 109 ( \pm 5) nm y espesor de $18( \pm 3) \mathrm{nm}$ (Leon et al., 2021). La Figuras 3(B) y 3(C) presentan el respectivo comportamiento potenciodinámico y potenciostático, tanto bajo condición de oscuridad como en iluminación, esto para los dos electrodos considerados: (i) $20 \%$ rutilo, (ii) $0 \%$ rutilo.

(A)

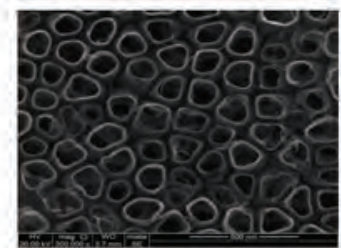

(B)

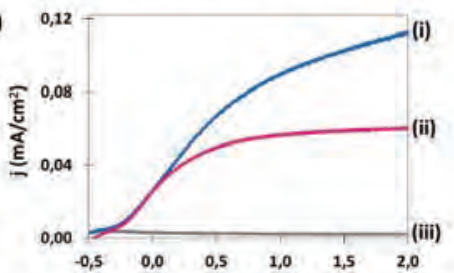

(c)

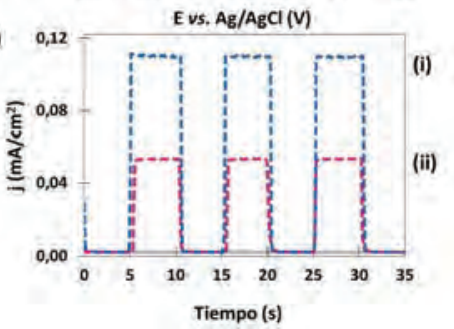

Figura 3. Vista frontal de arreglo de nanotubos de $\mathrm{TiO}_{2}$. Voltametrías de barrido lineal a $50 \mathrm{mV} / \mathrm{s}$ (B) y cronoamperometrías a $2,0 \mathrm{~V}$ vs. $\mathrm{Ag}-\mathrm{AgCl}$ (C): (i) $\mathbf{2 0} \%$ rutilo, (ii) 0 \% rutilo, (iii) oscuridad. (Leon et al., 2021) 
La Figura 4 presenta la respuesta fotoelectroquímica de los sistemas estudiados comparada con la corriente que resulta del modelo de Gärtner; siendo notorio el hecho de que, a elevados sobrepotenciales, las señales se aproximan asintóticamente al modelo representado por las ecuaciones 1 y 2 .

(A)

(B)
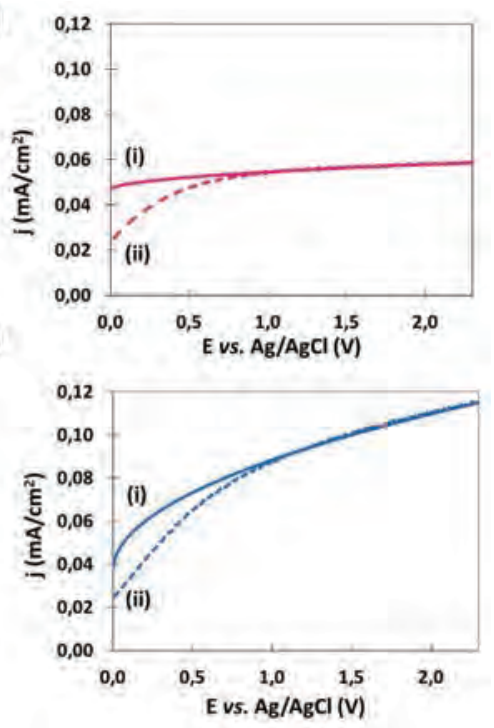

Figura 4. Respuesta fotoelectroquímica en relación al modelo de Gärtner. (i) Ec. 1 y (ii) Experimental. Electrodos con $0 \%$ (A) y $20 \%$ (B) de rutilo.

Con el objetivo de dejar en evidencia la existencia de estados superficiales, en la Figura 5 se presenta las voltametrías cíclicas en oscuridad de los electrodos considerados. El esquema energético de las bandas, $\mathrm{Fi}$ gura 5(A), supone que el borde inferior de la banda de conducción se corresponde con el potencial de banda plana de cada sistema, comparándose directamente con el eje de potencial de las voltametrías presentadas: Figuras 5(B) y 5(C). 

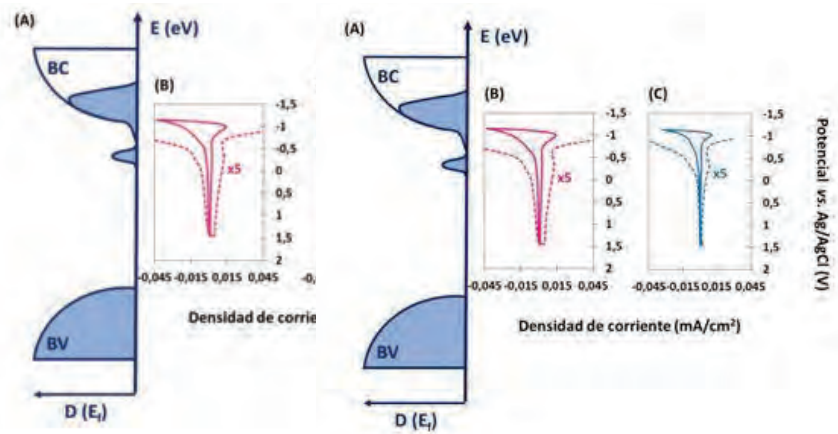

Figura 5. Interpretación energética de la respuesta de acumulación de cargas en oscuridad. Densidad de estados idealizada (A) y voltametrías cíclica en oscuridad de electrodos con $0 \%$ (B) y $20 \%$ (C) de rutilo. EBP determinado por Mott-Schottky (Ec. 3) resultó ser: -0.970 y $-0.935 \mathrm{~V}$ vs. $\mathrm{Ag}-\mathrm{AgCl}$ para $\mathrm{TiO}_{2} 0 \%$ y $20 \%$ rutilo, respectivamente.

Ahora bien, para estudiar los fenómenos de desactivación, la Figura 6 presenta la respuesta de fotoluminiscencia tanto estacionaria como resuelta en el tiempo. El espectro de emisión permite definir el intervalo energético de desactivación radiactiva (430 $800 \mathrm{~nm}$ ); mientras que los transitorios aportan información cinética. Estos últimos se analizaron mediante el método de ajuste con convolución, para ello se usaron los decaimientos exponenciales: $\mathrm{fi}(\mathrm{t})=A_{\mathrm{i}} \exp \left(-\mathrm{t} / \tau_{\mathrm{i}}\right), \mathrm{y}$ la señal de fondo: $g(t)$, para componer la función de intensidad de la señal luminosa:

$I(t)=\sum_{i=1}^{4} \int_{-\infty}^{\infty} g\left(t^{\prime}\right) f_{i}\left(t-t^{\prime}\right) d t^{\prime}$ (Wakabayashi et al., 2005). Las magnitudes que caracterizan los decaimientos son los factores pre-exponenciales $\left(A_{\mathrm{i}}\right)$ y los tiempos de vida $\left(\tau_{\mathrm{i}}\right)$, siendo estas últimas magnitudes que aportan información cinética del fenómeno de desactivación. 
(A)

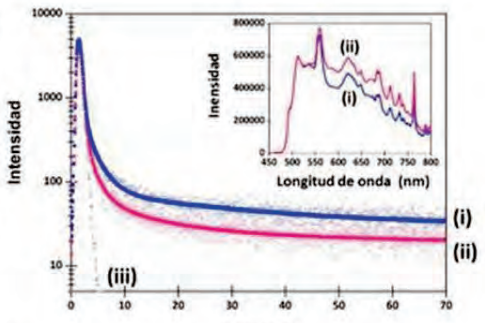

(B)

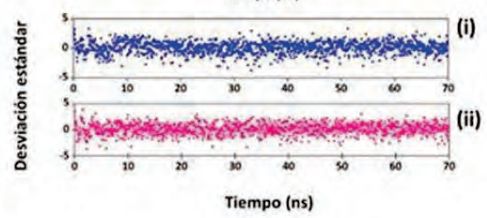

Figura 6. (A) Señal de fotoluminiscencia (emisión a $494 \mathrm{~nm}$ ) resuelta en el tiempo. Inserto: Espectros de emisión y (B) Residuales del ajuste al modelo de decaimiento tetra-exponencial. $\mathrm{TiO}_{2}$ con $20 \%$ (i), $0 \%$ (ii) de rutilo y blanco

(iii). Excitación a $296 \mathrm{~nm}$.

(Leon et al., 2021)
En la Tabla 1 se reportan los tiempos de vida $\left(\tau_{\dot{j}}\right)$ determinados para la desactivación de portadores de carga en los materiales sintetizados.

Tabla 1. Tiempos de vida de desactivación radiactiva y prueba CHI cuadrado $\left(\chi^{2}\right)$. Emisión a $494 \mathrm{~nm}$ y excitación a $296 \mathrm{~nm}$

\begin{tabular}{ccccc}
\hline $\begin{array}{c}\% \\
\text { rutilo }\end{array}$ & $\begin{array}{c}\tau_{1} \\
(\text { ns) }\end{array}$ & $\begin{array}{c}\tau_{2} \\
\text { (ns) }\end{array}$ & $\begin{array}{c}\tau_{3} \\
\text { (ns) }\end{array}$ & $\chi^{2}$ \\
\hline 0 & 2,25 & 26,86 & 2560 & 1,10 \\
20 & 1,86 & 10,22 & 0,153 & 1,14 \\
\hline
\end{tabular}

\section{DISCUSIÓN}

La respuesta fotoelectroquímica: Fotocorriente vs. potencial

Para ilustrar la respuesta fotoelectroquímica en nanotubos de $\mathrm{TiO}_{2}$ se realizaron voltametrías lineales a 50 $\mathrm{mV} / \mathrm{s}$. La Figura 3(B) presenta la respuesta obtenida para ambos electrodos bajo condiciones de ilumina ción continua y en oscuridad. Bajo iluminación continua se observa el aumento de la respuesta anódica, se- guido de un cambio de pendiente hasta establecerse el estado pseudoestacionario de la fotocorriente. Este aumento típico ocurre como consecuencia de la llegada de nuevos portadores de carga minoritarios hacia la interfase semiconductor | electrolito, donde participan en reacciones de transferencia con las especies donadoras en solución, en este caso $\mathrm{H}_{2} \mathrm{O}$ (Oliva et al., 2002). La cinética de oxidación del agua es una reac- 
ción electroquímica de múltiples pasos que puede resultar lenta en comparación con el tiempo de vida media de estos nuevos portadores fotogenerados, los cuales se pueden recombinar incluso antes de llegar a la interfase.

El aumento de fotocorriente observado no solo se asocia con los cambios en la cinética de transferencia, sino también con los cambios en la dinámica de transporte de estos portadores desde el seno del semiconductor hasta la interfase (Peter, 2013). En última instancia, es la concentración de los portadores en la interfase lo que determina el rendimiento del proceso fotoelectroquímico, y esta depende del balance dinámico entre los flujos de transferencia electrónica y de recombinación. Esta condición dinámica puede ser modulada a través del potencial aplicado, el cual es responsable del desdoblamiento de bandas, siendo la fuerza impulsora para el transporte de portadores hacia la interfase.

Los efectos por el desbalance de los flujos involucrados son evidentes en la condición de iluminación modulada, por ejemplo, ver la curva fotocorriente vs. tiempo en Figura 3(C).
$\mathrm{Al}$ interrumpirse el flujo de fotones incidentes, la fotocorriente del sistema decae hasta su condición de equilibrio (oscuridad), producto de la rápida desactivación de los portadores por recombinación. Al iluminarse nuevamente la superficie, ocurre rápidamente la separación del par electrón-hueco, lo cual lleva a que la concentración de estos portadores alcance el valor necesario para sostener la fotocorriente observada bajo iluminación continua.

La naturaleza del semiconductor también es otra variable capaz de modificar el balance entre estos flujos, debido a cambios en la dinámica de la interfase al introducirse nuevos estados electrónicos en el seno del material. Para ilustrar el efecto sobre la respuesta fotoelectroquímica al introducir una nueva fase en el semiconductor, se muestra la respuesta obtenida sobre un electrodo de nanotubos de $\mathrm{TiO}_{2}$ compuesto por $80 \%$ anatasa y $20 \%$ rutilo, bajo las mismas condiciones experimentales. Como se observa en las Figuras $3(\mathrm{~B})$ y $3(\mathrm{C})$, la fotocorriente obtenida es mayor en comparación con la del electrodo constituido por la fase anatasa. 
La nueva fase introduce defectos en el material que modifican sus propiedades eléctricas y ópticas, lo cual se manifiesta como una variación de la respuesta fotoelectroquímica. Bajo iluminación y condición de potencial aplicado los electrones son extraídos hacia el circuito externo, pero también pueden verse atrapados en nuevos estados superficiales introducidos por estos defectos (Qian et al., 2019). Vemos entonces cómo la respuesta fotoelectroquímica para estos materiales nanoestructurados se define por la condición energética impuesta y las propiedades características del semiconductor. Mientras que la primera puede modularse con el potencial aplicado, la segunda se ilustra al introducir una segunda fase.

Ante una dinámica que resulta compleja por la yuxtaposición de distintos fenómenos como la generación del par electrón-hueco, su recombinación, el alojamiento de los primeros en estados superficiales o la transferencia de los últimos en la interfase, surge la necesidad de establecer un estado de referencia que nos permita comentar sobre la contribución relativa de tales fenómenos y entender los límites naturales del sistema fotoelectroquímico.

\section{Límites naturales de la respuesta ex- perimental}

Bajo una interpretación netamente fenomenológica, los flujos de recombinación y transferencia determinan la cinética observada en la interfase semiconductor | electrolito. La máxima fotocorriente que puede ser colectada en el circuito externo se encuentra cuando todos los portadores de carga fotogenerados alcanzan la interfase y participan en la reacción redox, lo que significa que la energía absorbida por el material está siendo convertida. Esta condición en ausencia de recombinación está resumida en la Ec.1.

La respuesta de Gärtner representa la condición ideal bajo la cual se obtendría la máxima fotocorriente de un semiconductor en estudio, por lo que la comparación de la respuesta experimental con este modelo resulta determinante para evidenciar las pérdidas en la colección de portadores en la interfase. En la Figura 4 se puede observar que solo a altos sobrepotenciales se alcanza tal desempeño ideal, donde el flujo de transferencia predomina frente al de recombinación. A expensas de introducir trabajo eléctrico para la extrac- 
ción de los electrones, se define una condición experimental que busca aproximarse a uno de los límites de respuesta para elucidar efectos debidos a la naturaleza de los electrodos.

Las diferencias intrínsecas entre las distintas fases no sólo se interpretan como un aumento de la fotocorriente del sistema, sino también como una mejora en la colección de electrones debido a la minimización de la recombinación, lo que resulta en una mejor aproximación al desempeño ideal a elevados sobrepotenciales. En el otro extremo, el proceso está limitado por una condición donde el flujo de recombinación es determinante frente al de transferencia electrónica, resolviéndose una menor diferencia entre la curva experimental y la de Gärtner para el electrodo con $80 \%$ anatasa y $20 \%$ rutilo.

Ante efectos que resultan complementarios, surge la necesidad de una caracterización dinámica de la interfase con diferentes técnicas que permitan desacoplar fenómenos que ocurren a diversas frecuencias. Por ejemplo, a través de la espectroscopia de fotocorriente de intensidad modulada (IMPS, por sus siglas en inglés), se puede modificar la perturbación luminosa al sistema para entrar en frecuencia con fenómenos que ocurren a diferentes escalas de tiempo. En general, esta técnica consiste en perturbar la intensidad de luz de una forma sinusoidal, permitiendo medir la fase y magnitud de la función de transferencia del sistema a diferentes frecuencias. A través de los resultados obtenidos por esta perturbación se pueden cuantificar los flujos de transferencia de carga y de recombinación con sus constantes características (Peter et al., 1997; Peter, 2013).

Cabe destacar que los métodos espectroscópicos también surgen Como técnicas fundamentales para abordar la separación de fenómenos, y, sabiendo que los sistemas nanoestructurados presentan acumulación de carga en estados superficiales, resulta conveniente usar métodos espectroscópicos que den cuenta de la desactivación radiactiva de los electrones fotoexcitados en su decaimiento hacia la banda de valencia. La espectroscopia de fotoluminiscencia estacionaria permite determinar la zona de emisión en función 
de la energía de excitación, y la adquisición de la señal resuelta en el tiempo permite estimar los tiempos de vida de los estados emisores (Smandek et al., 1989; Gilliland, 1991). En todo caso, luego de tener una evidencia de la existencia de estados superficiales, resulta interesante estimar las escalas tiempo de los procesos que derivan en recombinación de portadores.

Una primera aproximación para probar la existencia de estados la proproporcionaron los integrantes del grupo de Bisquert (Bisquert, 2003; Fabregat-Santiago et al., 2003; MonIlor-Satoca et al., 2020), quienes fueron los primeros en mostrar las potencialidades de la voltametría cíclica para este propósito. Las corrientes capacitivas medidas pueden atribuirse al Ilenado / vaciado de estados electrónicos. La idea es que la corriente capacitiva sea proporcional a la variación del número de estados en función del potencial.

Ahora bien, para $\mathrm{TiO}_{2}$, la ubicación de estos estados corresponde a: (i) la banda de conducción, (ii) a una distribución exponencial de estados de superficie por debajo de la BC y, (iii) en ciertas ocasiones, una modificación localizada de la densidad de estado resulta en la aparición de los estados energéticos llamados profundos (Berger et al., 2012).

La Figura 5 muestra las voltametrías cíclicas de los sistemas estudiados, proporcionando una interpretación energética de la respuesta de acumulación de cargas en oscuridad. La señal amplificada (x5) se representa hasta el valor respectivo de EBP para cada electrodo, permitiendo delimitar los estados de banda de conducción de los estados superficiales y de los profundos. De lo anterior resulta posible aseverar que los electrones excitados podrían participar en una dinámica de carga y descarga de estos estados energéticos, por lo que estudiar su desactivación radiativa promete un camino para profundizar en los procesos de recombinación.

Como se comentó a partir de la Figura 5, cambios intrínsecos como la fase en el material pueden llevar a cambios en la dinámica de los portadores; entonces, a partir de una condición inicial con excitación de energía superior a la brecha de bandas, se puede determinar la emisión 
o desactivación de electrones desde estados de menor energía inferiores a la banda de conducción.

La Figura 6(A) muestra los decaimientos de la señal de fotoluminiscencia a circuito abierto, a partir de una perturbación luminosa seguida de la restitución a la condición de equilibrio. En el inserto se presentan los espectros de emisión de los dos electrodos bajo estudio.

En general, se observa señal de emisión en toda la región visible, indicando la existencia de estados de energía menor a EBC. Se destaca que el intervalo de emisión de las señales obtenidas es consistente con el espectro de emisión reportado para nanoestructuras de $\mathrm{TiO}_{2}$ (Yurdakal et al., 2019). El espectro permite confirmar que la incorporación de rutilo trae como consecuencia que la señal tenga menor intensidad, lo que se puede asociar a menor cantidad de eventos de recombinación, ya que los portadores estarían participando en procesos redox en la interfase.

La señal de los decaimientos es sostenida por la supervivencia de los portadores generados con la pertur- bación inicial, lo que permite cuantificar sus tiempos de vida $\left(\tau_{\mathrm{j}}\right)$, los cuales son magnitudes inversamente proporcionales a las constantes cinéticas de recombinación. Para los electrodos de $\mathrm{TiO}_{2}$ nanoestructurados las señales de decaimiento de emisión se ajustan a una función tetra-exponencial, lo que sugiere la presencia de tres especies emisoras diferentes y una muy rápida atribuida a la respuesta del instrumental de fondo o blanco. La Figura 6(B) muestra los residuales de los decaimientos de la señal de fotoluminiscencia a circuito abierto representados en la Figura 6(A). Las tres constantes de tiempo fenomenológicas que resultan del análisis de la señal se atribuyen a la desactivación radiactiva de electrones en la banda de conducción $\left(\tau_{1}\right)$, estados de superficie $\left(\tau_{2}\right)$ y estados profundos $\left(\tau_{3}\right)$, respectivamente (Madriz et al., 2020; Leon et al., 2021). Los valores se presentan en la Tabla 1 resumiéndose que, la presencia de rutilo afecta los procesos de recombinación y especialmente los asociados con estados de menor energía. La población relativa de estados electrónicos y su dinámica se ve afectada, lo que resulta en una respuesta global con efectos 
de convolución. Las consecuencias y aplicaciones de estos resultados en los mismos sistemas se podrán consultar en la literatura (Leon et al., 2021). En todo caso, el desempeño de un sistema fotoelectroquímico se encuentra relacionado a la eficiencia de conversión de energía, y al menos la condición de máxima potencia resulta conveniente para la evaluación de su funcionalidad actual en relación a aplicaciones interesantes (Bao et al., 2012; Pellet et al., 2019; Fountaine et al., 2020; Monllor-Satoca et al., 2020; Madriz et al., 2020).

El grupo de Gerischer demostró que al mismo potencial $(E)$ y dada una eficiencia de recombinación constante $(k)$, la intensidad de densidad de fotocorriente $\left(V_{\mathrm{FC}}\right)$ se relaciona con la intensidad de fotoluminiscencia $\left(I_{\mathrm{FL}}\right)$ según la ecuación 4; es decir, mayor fotocorriente, menor luminiscencia (Smandek et al., 1989). Por tanto, los cambios relativos observados en los sistemas de estudio dejan en evidencia la relación cualitativa entre los flujos de transferencia y recombinación, siendo esta última dominante a bajos sobrepotenciales.

$$
\frac{J_{F C(E)}}{I_{0}}=1-\frac{I_{F L(E)}}{k I_{0}}
$$

En los términos planteados a lo largo del presente trabajo se resaltará que el límite inferior del proceso fotoelectroquímico está dominado por el flujo de recombinación, donde toda la energía se disipa por procesos de desactivación. Mientras que la máxima fotocorriente está definida cuando el flujo de transferencia de electrones se puede colectar con la máxima eficiencia; es decir, a elevados sobrepotenciales donde la recombinación se ha minimizado totalmente. Estos son los límites naturales del proceso, y explorarlos permitirá elucidar diferencias fundamentales en electrodos nanoestructurados, los cuales presenten alguna modificación interesante que impacte sobre la densidad de estados.

\section{CONCLUSIÓN}

Frente al hecho de que diferentes electrodos semiconductores se pue- den interpretar con una fenomenología similar, explorar las limitacio- 
nes naturales del proceso fotoelectroquímico es determinante para elucidar la contribución relativa de los fenómenos conducentes: transferencia electrónica y recombinación de portadores. Entender la recombinación a través de técnicas dinámicas es fundamental para elucidar su papel en nanoestructuras.

\section{AGRADECIMIENTOS}

Al Grupo de Electroquímica de la Universidad Simón Bolívar por las estimulantes discusiones sobre la temática desarrollada. Agradecemos a The World Academy of Sciences (TWAS) and the TWAS Young Affiliates Network (TYAN), por generar las condiciones necesarias para catalizar la cooperación científica entre los Doctores LM, FMC y RV.

\section{LISTA DE REFERENCIAS}

Bao, N., Feng, X., \& Grimes, C. (2012). Self-organized one-dimensional $\mathrm{TiO}_{2}$ nanotube/nanowire array films for use in excitonic solar cells: A review. Journal of Nanotechnology, Volume 2012, 645931.

Berger, T., Monllor-Satoca, D., Jankulovska, M., Lana-Villareal, T., \& Gómez, R. (2012). The electrochemistry of nanostructured titanium dioxide electrodes. ChemPhysChem, 13,2824-2875.

Bertoluzzi, L., Badia-Bou, L., Fabregat-Santiago, F., Gimenez, S., \& Bisquert, J. (2013). Interpretation of cyclic voltammetry measurements of thin semiconductor films for solar fuel applications. Journal of Physical Chemistry Letters, 4(8),1334-1339.

Bisquert, J. (2003). Chemical capacitance of nanoestructured semiconductors: its origin and significance for nanocomposite solar cells. Physical Chemistry Chemical Physics, 5, 5360-5364.

Bisquert, J. (2017). Nanostructured energy devices: Foundations of carrier transport. Boca Raton: CRC Press. Taylor \& Francis Group. 
Fabregat-Santiago, F., Mora-Seró, I., García-Belmonte, G., \& Bisquert, J. (2003). Cyclicvoltammetry studies of nanoporous semiconductors. Capacitive and reactive properties of nanocrystalline $\mathrm{TiO}_{2}$ electrodes in aqueous electrolyte. Journal of Physical Chemistry B, 107, 758-768.

Fountaine, K., Lewerenz, H., \& Atwater, H. (2020). Efficiency limits for photoelectrochemical water-splitting. Nature Communications, 7, 13706.

Garcia-Segura, S., \& Brillas, E. (2017). Applied photoelectrocatalysis on the degradation of organic pollutants in wastewaters. Journal of Photochemistry and Photobiology C: Photochemistry Reviews, 31, 1-35.

Gärtner, W. (1959). Depletion-layer photoeffects in semiconductors. Physical Review, 116(1), 84-89.

Gerischer, H. (1990). The impact of semiconductors on the concepts of electrochemistry. Electrochimica Acta, 35(11-12), 1677-1699.

Gilliland, G.D. (1997). Photoluminescence spectroscopy of crystalline semiconductors. Materials Science and Engineering, R18, 99-400.

Gutiérrez, M., Martín, C., Souza, B.E.,Van der Auweraer, M., Hofkens, J., \& Tan, J.C. (2020) Highly luminescent silver-based MOFs: Scalable eco-friendly synthesis paving the way for photonics sensors and electroluminescent devices. Applied Materials Today, 21, 100817.

He, Y., Chen, R., Fa, W., Zhang, B., \& Wang, D. (2019) Surface chemistry and photoelectrochemistry-Case study on tantalum nitride. The Journal of Chemical Physics, 151, 130902(1-11).

Kim, J.Y., Lee, J.-W., Jung, H.S., Shin, H., \& Park., N.G.(2020). High-Efficiency perovskite solar cells. Chemical Reviews, 120, 7867-7918.

Kumaravel, V., Mathew, S., Bartlett, J., \& Pillai, S. C. (2019). Photocatalytic hydrogen production using doped $\mathrm{TiO}_{2}$ : A review of recent advances. Applied Catalysis $B$ : Environmental, 244, 1021-1064. 
Kokkinos, C.,\& Economou, A.(2020). Recent advances in voltammetric, amperometric and ion-selective (bio)sensors fabricated by microengineering manufacturing approaches. Current Opinion in Electrochemistry, 23 21-25.

Lee, K., Mazare, A., \& Schmuki, P. (2014). One-dimensional titanium dioxide nanomaterials: Nanotubes. Chemical Reviews, 114 (19), 9385-9454.

Leon, D. (2020). Comportamiento electroquímico de nanotubos de $\mathrm{TiO}_{2}$ : relación de fases y fotooxidación de p-nitrofenol. Dissertation, (pp. 1-102). Universidad Simón Bolívar, Caracas, Venezuela.

Leon, D., Maimone, A., Carvajal, D., Madriz, L., Scharifker, B. R., Cabrerizo, F.M. \& Vargas, R. (2021). Unraveling kinetic effects during photoelectrochemical mineralization of phenols. Rutile:Anatase $\mathrm{TiO}_{2}$ nanotubes photoanodes in thin-layer condition. Journal of Physical Chemistry C, 125(1), 610-617.

Lianos, P. (2017). Review of recent trends in photoelectrocatalytic conversion of solar energy to electricity and hydrogen. Applied Catalysis B: Environmental, 210, 235254.

Madriz, L., Tatá, J., Carvajal,D., Núñez, O., Scharifker, B.R., Mostany, J., Borrás, C., Cabrerizo, F.M., \& Vargas, R. (2020). Photocatalysis and photoelectrochemical glucose oxidation on $\mathrm{Bi}_{2} \mathrm{WO}_{6}$ : conditions for the concomitant $\mathrm{H}_{2}$ production. Renewable Energy, 152, 974-983.

Maimone, A. (2018). Relación anatasa-rutilo en nanotubos de TiO2: Aspectos termodinámicos y fotoelectroquímicos. Master Thesis, (pp. 1-163). Universidad Simón Bolívar, Caracas, Venezuela.

Maimone, A., Camero, S., \& Blanco, S., (2015). Caracterización del óxido de titanio obtenido mediante tratamiento térmico y anodizado electroquímico. Revista de la Facultad de Ingeniería U.C.V, 30, 189-200.

Memming, R. (2015). Semiconductor electrochemistry. Rellingen: Wiley-VCH.

Monllor-Satoca, D., Díez-García, M. I., Lana-Villareal, T., \& Gómez, R. (2020). Photoelectrocatalytic production of solar fuels with semiconductor oxides: materials, activity and modeling. Chemical Communications, 56, 12272-12289. 
Naranjo, D. I., García-Vergara, S. J., \& Blanco, S. (2017). Scanning electron microscopy of heat treated $\mathrm{TiO}_{2}$ nanotubes arrays obtained by anodic oxidation. Journal of Physics: Conference Series, 935, 012025.

Nevaréz-Martínez, M.C., Mazierki, P., Kobylanski, M.P., Szczepanska, G., Trykowski, G., Malankowska, A., Kozaz, M., Espinoza-Montero, P., \& Zaleska-Medynska, A. (2018). Growth, structure and photocatalytic properties of hierarchical V2O5$\mathrm{TiO}_{2}$ nanotube arrays obtained from the one-step anodic oxidation of Ti- $\mathrm{V}$ alloys. Molecules, 22, 580.

Oliva, F.Y., Avalle, L.B., Santos, E., \& Cámara, O.R., (2002). Photoelectrochemical characterization of nanocrystalline $\mathrm{TiO}_{2}$ films on titanium substrates, Journal of Photochemistry and Photobiology A: Chemistry, 146, 175-188.

Pellet, N., Giordano, F., Zakeeruddin, S, M. \& Grätzel, M. (2019). Device and method for performing maximum power point tracking for photovoltaic devices in presence of hysteresis. US Patent, 10, 488, 879.

Peter, L. (2013). Kinetics and mechanisms of light-driven reactions at semiconductor electrodes: Principles and techniques. En: Lewerenz. H-J., \& Peter. L. (Eds.). Photoelectrochemical Water Spliting. Materials, processes and architectures. (pp. 1951). Cambridge: RSC Energy \& Environmental Series.

Peter, L., Gurudayal., Wong, L. H., \& Abdi, F. F. (2018). Understanding the role of nanoestructuring in photoelectrode performance for light-driven water splitting. Journal of Electroanalytical Chemistry, 819,447-458.

Peter, L. M., Ponomarev, E. A., \& Fermín, D. J. (1997). Intensity-modulated photocurrent spectroscopy: Reconciliation of phenomenological analysis with multistep electron transfer mechanisms. Journal of Electroanalytical Chemistry, 427(1-2), 7996.

Qian, R., Zong, H., Schneider, J., Zhou, G., Zhao, T., Li, Y., Yang, J., Bahnemann, D.W., \& Pan,J.(2019). Charge carrier trapping, recombination and transfer during $\mathrm{TiO}_{2}$ photocatalysis: An overview. Catalysis. Today, 335, 78-90. 
Reichman, J. (1980). The current-voltage characteristics of semiconductors-electrolyte junction photovoltaic cells. Applied Physics Letters,36(7), 574-577.

Rueda, H., Becerra, J., \& Blanco, S. (2018). Effect of the oxygen diffusion in the anatase-rutile transformation in a $\mathrm{TiO}_{2}$ nanotubes array obtained by electrochemical anodization. Journal of Physics: Conference Series, 1119, 012026 (1-6).

Sato, N. (1998). Electrochemistry at metal and semiconductor electrodes. Richmond: Elsevier Science B. V.

Smandek, B., Chmiel, G., \& Gerischer, H., (1989). Photoluminescence as an in-situ technique to determine solid state and surface properties of semiconductors in an electrochemical cell-application of the "Dead Layer Model". Berichte der Bunsengesellschaft für physikalische Chemie, 93, 1094-1103.

Vargas, R., Carvajal, D., Galavis, B., Maimone, A., Madriz, L., \& Scharifker, B.R. (2019). High-field growth of semiconducting anodic oxide films on metal surfaces for photocatalytic applications. International Journal of Photoenergy, vol.2019, (15pp).

Vargas, R., Carvajal, D., Madriz, L., \& Scharifker, B. R. (2020). Chemical kinetics in solar to chemical energy conversion: the photoelectrochemical oxygen transfer reaction. Energy Reports, 6,2-12.

Wakabayashi, K., Yamaguchi, Y., Sekiya, T., \& Kurita, S. (2005). Time-resolved luminescence spectra in colorless anatase $\mathrm{TiO}_{2}$ single crystal. Journal of Luminescense, $112,50-53$.

Yurdakal, S., Garlisi, C., Ozcan, L., Bellardita, M., \& Palmisano, G., (2019). (Photo)catalysis characterization techniques: Adsorption isotherms and BET, SEM, FTIR, UV-Vis, Photoluminescence and Electrochemical characterizations. In: Marcí, G.\& Palmisano, L., (Eds.). Heterogeneous photocatalysis. Relationships with heterogeneous catalysis and perspectives (pp. 87-152). Amsterdam: Elsevier.

Zhang, Q., Celorio, V., Bradley, K., Eisner, F., Cherns, D., Yan, E., \& Fermín, D. (2014). Density of deep trap states in oriented $\mathrm{TiO}_{2}$ nanotube arrays. Journal of Physical Chemistry C, 118(31), 18207-18213. 